\title{
Hospitalization Rates in Patients with Heart Failure and Reduced Ejection Fraction Initiating Sacubitril/ Valsartan or Angiotensin-Converting Enzyme Inhibitors/Angiotensin Receptor Blockers: A Retrospective Cohort Study
}

\author{
Emma Houchen · Emil Loefroth · Raymond Schlienger · Clare Proudfoot • \\ Stefano Corda $\cdot$ Sibasish Saha $\cdot$ Sarvesh K. Satwase $\cdot$ Rachel Studer
}

Received: November 17, 2021 / Accepted: December 27, 2021 / Published online: January 29, 2022

(C) The Author(s) 2022

\section{ABSTRACT}

Introduction: The angiotensin receptor neprilysin inhibitor (ARNI) sacubitril/valsartan (SAC/ VAL) has shown benefit in patients with symptomatic heart failure (HF), including those naïve to renin-angiotensin-aldosterone system inhibitor (RAASi) therapy, and is considered the preferred RAASi for chronic HF. Real-world data on ARNI, specifically in RAASi-naïve patients, are limited. This study compared real-world outcomes of ARNI (SAC/VAL) vs. angiotensinconverting enzyme inhibitor (ACEi) or angiotensin receptor blocker (ARB) therapy in RAASinaïve patients with $\mathrm{HF}$ and reduced ejection fraction (HFrEF).

Methods: This retrospective cohort study included de-identified data on RAASi-naïve

E. Houchen $(\bowtie)$

Novartis Ireland Ltd., Vista Building, Elm Park, Merrion Road, Dublin 4 DO4A9N6, Ireland e-mail: emma.houchen@novartis.com

\section{E. Loefroth}

Novartis Sweden AB, Kista, Sweden

R. Schlienger - C. Proudfoot · S. Corda - R. Studer Novartis Pharma AG, Basel, Switzerland

S. Saha $\cdot$ S. K. Satwase

Novartis Healthcare Pvt. Ltd, Hyderabad, India

E. Houchen

Medical and Knowledge Solutions, Novartis Global Service Center, Dublin, Ireland patients with HFrEF (left ventricular ejection fraction $\leq 40 \%$ ) who had newly initiated SAC/ VAL or ACEi/ARB between July 1, 2015, and March 31, 2019, from the Optum ${ }^{\circledR}$ Electronic Health Records database in the US. New SAC/ VAL users were propensity score matched 1:2 with new ACEi/ARB users by pre-selected characteristics. One-year post-index rates of allcause, HF, and cardiovascular hospitalizations and the composite of $\mathrm{HF}$ hospitalization or emergency room (ER) visits were measured using negative binomial regression. Time to first all-cause hospitalization, HF hospitalization, and composite of HF hospitalization or ER visits was measured using a subdistribution hazards model.

Results: The matched sample included 3059 new SAC/VAL and 6118 new ACEi/ARB users. Rates of all-cause hospitalization and composite of HF hospitalization or ER visits were significantly lower with SAC/VAL compared with ACEi/ARB (incidence rate ratio [95\% confidence interval]: 0.87 [0.81-0.93] and 0.87 [0.81-0.94], respectively), whereas rates of HF hospitalizations and cardiovascular hospitalizations were similar (1.00 [0.91-1.11] and 0.94 [0.87-1.02], respectively). Time-to-event analyses also showed a similar trend.

Conclusions: In real-world clinical practice, RAASi-naïve patients with HFrEF initiating SAC/ VAL were less likely to be hospitalized than those initiating ACEi/ARB, suggesting a potential for a reduced clinical and economic burden 
in these patients.Keywords: Angiotensin-converting enzyme inhibitor; Angiotensin receptor blocker; Angiotensin receptor neprilysin inhibitor; Heart failure; Sacubitril/valsartan

\section{Key Summary Points}

\section{Why carry out this study?}

There is a scarcity of real-world outcomes data in patients with heart failure and reduced ejection fraction (HFrEF) initiating an angiotensin receptor neprilysin inhibitor (ARNI) who have not previously received a renin-angiotensin-aldosterone system inhibitor (RAASi).

This is the first study that specifically focuses on RAASi-naïve patients with HFrEF and demonstrated the benefit of an ARNI (sacubitril/valsartan [SAC/VAL]) compared with traditional first-line therapies (angiotensin-converting enzyme inhibitors [ACEi]/angiotensin receptor blockers $[\mathrm{ARB}])$ in a real-world setting.

\section{What was learned from the study?}

The results of the present real-world study demonstrate the clinical benefit of directly initiating SAC/VAL rather than traditional first-line therapies, such as ACEi/ARB, in patients with HFrEF.

The benefit of sacubitril/valsartan (SAC/ VAL) over ACEi/ARB in RAASi-naïve patients with HFrEF observed in this study validates the findings of a randomized controlled trial, PIONEER-HF, where SAC/ VAL was found to be superior to enalapril in a subgroup of RAASi-naïve patients.

In patients with HFrEF naïve to RAAS inhibition, healthcare providers need to consider the benefit of initiating ARNI in reducing all-cause hospitalizations, thereby potentially reducing the overall disease burden.

\section{INTRODUCTION}

Heart failure (HF) affects approximately 6 million adults in the United States (US) [1], and its prevalence is projected to increase by $15 \%$ globally and by $23 \%$ in the US by 2030 [2]. The renin-angiotensin-aldosterone system (RAAS) inhibition is the cornerstone of therapy for HF with reduced ejection fraction (HFrEF; left ventricular ejection fraction $[\mathrm{LVEF}] \leq 40 \%$ ), which has traditionally included angiotensin-converting enzyme inhibitors (ACEi) and angiotensin receptor blockers (ARB) [3, 4]. Sacubitril/valsar$\tan (\mathrm{SAC} / \mathrm{VAL})$ is a first-in-class angiotensin receptor neprilysin inhibitor (ARNI), which simultaneously delivers RAAS inhibition through valsartan and neprilysin inhibition through sacubitril. SAC/VAL was first approved by the US Food and Drug Administration in July 2015 for the treatment of chronic HFrEF [5]. The latest update of the American College of Cardiology (ACC) Expert Consensus Decision Pathway (ECDP) on HF treatment recommends ARNI as the preferred RAAS inhibitor (RAASi) for HFrEF in patients who have been previously treated with an ACEi or ARB, as well as in patients naïve to an ACEi or ARB [6]. SAC/VAL has shown superiority over enalapril in reducing all-cause mortality and HF hospitalization in patients with HFrEF and New York Heart Association (NYHA) class II-IV who had been previously treated with an ACEi/ARB in the PARADIGM-HF trial [7], and in patients hospitalized with acute decompensated HF in the PIONEER-HF trial [8-10]. Moreover, there is increasing evidence of the effectiveness and safety of SAC/VAL in real-world clinical practice [11-17]. Subgroup analyses of clinical trials, including PIONEER-HF [10] and PROVE-HF [18], have shown a consistent benefit of SAC/VAL in ACEi/ARB-naïve patients as in those with prior exposure. This is complemented by data showing consistent safety and tolerability in ACEi/ ARB-naïve patients from PIONEER-HF [10] and TRANSITION-HF [19]. However, there are limited data on this patient population in the realworld setting. Because patients with HF who are naïve to RAAS inhibition may differ from those with prior exposure in terms of baseline 
characteristics and tolerability to new treatments, it is of interest to evaluate the real-world effectiveness of SAC/VAL specifically in patients naïve to RAAS inhibition. Therefore, we aimed to compare the hospitalization rates and time to hospitalization between RAASi-naïve patients with HFrEF initiating SAC/VAL and those initiating traditional first-line ACEi/ARB therapy, using data from the US Optum ${ }^{\circledR}$ Electronic Health Records (EHR) database.

\section{METHODS}

\section{Study Design and Data Sources}

This retrospective cohort study was conducted using secondary data from a large US EHR database provided by OptumLabs spanning the period from July 1, 2014, to December 31, 2019 (Fig. 1). The Optum EHR database contains deidentified and aggregated clinical medical administrative data from 85 US healthcare delivery organizations spread across 50 states. Clinical and other medical administrative data available from the Optum database were obtained from both inpatient and ambulatory EHRs, practice management systems, and numerous other internal systems. Institutional review board approval was deemed unnecessary because the information retrieved from the database was de-identified.

\section{Patient Identification and Cohort Assignment}

The study included adult patients ( $\geq 18$ years of age) with at least one International Classification of Diseases-9-Clinical Modification (ICD-9$\mathrm{CM}$ ) or ICD-10-CM code for a diagnosis of HF within 1 year prior to the index date. Eligible patients were required to have an LVEF of $\leq 40 \%$ at the latest assessment in the pre-index period and an initial prescription of SAC/VAL or ACEi/ARB during the identification period (i.e., between July 1, 2015, and March 31, 2019). The ICD-9-CM and ICD-10-CM codes used for the diagnosis of $\mathrm{HF}$ are provided in Table 1 . SAC/ VAL and ACEi/ARB could be prescribed in either inpatient or outpatient settings. Patients were required to be naïve (without prescription) to both SAC/VAL and ACEi/ARB treatments for 365 days before the index date. The index date was defined as the first date of prescription of SAC/VAL or ACEi/ARB during the identification period. Patients with a first-time SAC/VAL prescription and no ACEi/ARB prescription in the 365 days preceding the SAC/VAL index date were assigned to the SAC/VAL cohort (new SAC/ VAL users), and patients with a first-time ACEi/ ARB prescription and no ACEi/ARB prescription in the 365 days preceding the ACEi/ARB index date were assigned to the ACEi/ARB cohort (new ACEi/ARB users).

Because information on continuous enrollment in the health plan was not available from the database, a proxy measure was used to identify patients who had their first date being active in a plan was 365 days before the index

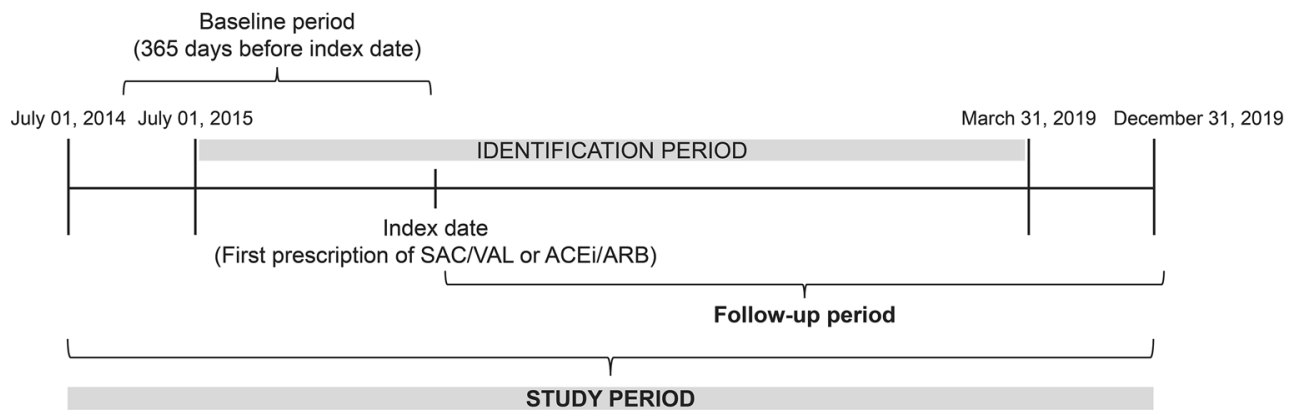

Fig. 1 Study design. $S A C / V A L$ sacubitril/valsartan, $A C E i$ angiotensin-converting enzyme inhibitor, $A R B$ angiotensin receptor blocker 
Table 1 ICD-9-CM and ICD-10-CM codes used for HF diagnosis

\begin{tabular}{llc}
\hline Disease & ICD-9-CM & ICD-10-CM \\
\hline HF & 398.91, 402.01, 402.11, 402.91, 404.01, 404.03, & I09.81, I11.0, I13.0, I13.2, I50, I50.1, I50.2, I50.20, \\
& $404.11,404.13,404.91,404.93,428,428.0$, & I50.21, I50.22, I50.23, I50.3, I50.30, I50.31, \\
& $428.1,428.2,428.20,428.21,428.22,428.23$, & I50.32, I50.33, I50.4, I50.40, I50.41, I50.42, \\
& $428.3,428.30,428.31,428.32,428.33,428.4$, & I50.43, I50.8, I50.81, I50.810, I50.811, I50.812, \\
& $428.40,428.41,428.42,428.43,428.9$ & I50.813, I50.814, I50.82, I50.83, I50.84, I50.89, \\
& & I50.9, I97.13, I97.130, I97.131 \\
Cardiomyopathy & $674.54,674.53,674.52,674.51,674.50,674.5$, & A36.81, B33.24, I25.5, I42, I I42.0, I42.1, I 42.2, \\
& $425.9,425.8,425.7,425.5,425.2,425.18,425.11$, & I42.5, I42.6, I42.7, I 42.9, I 43, O90.3 \\
& $425,425.1$ & \\
\hline
\end{tabular}

$H F$ heart failure, ICD International Classification of Diseases, $C M$ Clinical Modification

date and last date being active in a plan was on or beyond the index date. Patients were followed until December 31, 2019 (end of study), death, or health plan disenrollment. Patients were also required to have no missing data for sex and year of birth. Furthermore, patients who had no valid SAC/VAL prescription on the index or 365 days before the index date and were subsequently prescribed SAC/VAL were excluded from the new ACEi/ARB cohort to keep the two cohorts mutually exclusive.

\section{Outcomes}

The primary endpoint assessed in the study was the rate of HF hospitalizations for both new SAC/VAL users and new ACEi/ARB users during the post-index period. Secondary endpoints included the rate of the composite of HF hospitalization or emergency room (ER) visits, rate of all-cause hospitalizations, rate of cardiovascular hospitalizations, time to first HF hospitalization, time to first HF hospitalization or ER visit, and time to first all-cause hospitalization for new SAC/VAL users and new ACEi/ARB users during the post-index period. HF hospitalizations and HF ER visits were defined per ICD-9CM and ICD-10-CM codes for HF listed within the primary diagnoses associated with a hospitalization or an ER visit, respectively, that occurred during the follow-up period. All-cause hospitalizations were defined as any hospitalization that occurred during the followup period.

\section{Statistical Methods}

A feasibility analysis conducted in the Optum EHR database identified 1453 adults with HFrEF between July 1, 2015, and June 30, 2018, with at least 1 year of follow-up from the initial SAC/ VAL prescription and naive to SAC/VAL, ACEi, and ARB for at least 1 year prior to the index date. It was estimated that the study would be able to detect a response rate ratio of $<0.86$ or $>1.16$ using an anticipated event rate of 0.249 (lowest hospitalization rate reported), a sample size of 1400 , a mean exposure time of 1 year, and an over-dispersion parameter of 1.0, to generate results with 0.8 (1-beta) power at a 0.05 (two-sided alpha) significance level.

The new SAC/VAL users and new ACEi/ARB users were propensity score matched 1:2 using a greedy many-to-one algorithm to account for potential bias and confounding. The patients were matched on selected demographics and pre-index baseline characteristics, including age; sex; year of index date; race; ethnicity; geographical region; Elixhauser Comorbidity Index (ECI); comorbidities; signs or symptoms; LVEF; background medications; and previous all-cause hospitalization, HF hospitalization, HF-specific outpatient visit, and HF-specific ER visit in the preceding year. Unmatched patients 
Table 2 Demographic characteristics of patients before and after propensity score matching

\begin{tabular}{|c|c|c|c|c|c|c|c|c|}
\hline \multirow[t]{2}{*}{ Characteristics } & \multicolumn{4}{|c|}{ Before propensity score matching } & \multicolumn{4}{|c|}{ After propensity score matching } \\
\hline & $\begin{array}{l}\text { New SAC/VAL } \\
\text { users } \\
(N=3367)\end{array}$ & $\begin{array}{l}\text { New ACEi/ARB } \\
\text { users } \\
(N=\mathbf{5 0 , 8 7 2 )}\end{array}$ & $\begin{array}{l}\text { SMD } \\
(\%)\end{array}$ & $P$ value & $\begin{array}{l}\text { New SAC/VAL } \\
\text { users } \\
(N=3059)\end{array}$ & $\begin{array}{l}\text { New ACEi/ } \\
\text { ARB users } \\
(N=6118)\end{array}$ & $\begin{array}{l}\text { SMD } \\
(\%)\end{array}$ & $P$ value \\
\hline $\begin{array}{l}\text { Age (years) at } \\
\text { index, mean } \\
\text { (SD) }\end{array}$ & $65.92(13.08)$ & $66.94(13.74)$ & 7.64 & $<0.0001$ & $66.02(13.08)$ & $66.18(14.01)$ & 1.20 & 0.19 \\
\hline \multicolumn{9}{|l|}{ Sex, $n(\%)$} \\
\hline Female & $967(28.72)$ & $17,927(35.24)$ & 14.01 & $<0.0001$ & $909(29.72)$ & $1829(29.90)$ & 0.39 & 0.86 \\
\hline Male & $2400(71.28)$ & $32,945(64.76)$ & 14.01 & & $2150(70.28)$ & $4289(70.10)$ & 0.39 & \\
\hline \multicolumn{9}{|c|}{ Year of index, $n(\%)$} \\
\hline 2015 & $92(2.73)$ & $8505(16.72)$ & 48.57 & $<0.0001$ & $92(3.01)$ & $198(3.24)$ & 1.32 & 0.92 \\
\hline 2016 & $638(18.95)$ & $18,140(35.66)$ & 38.18 & & $636(20.79)$ & $1291(21.10)$ & 0.76 & \\
\hline 2017 & $1040(30.89)$ & $12,688(24.94)$ & 13.29 & & $991(32.40)$ & $1980(32.36)$ & 0.07 & \\
\hline 2018 & $1239(36.80)$ & $9377(18.43)$ & 41.97 & & $1056(34.52)$ & $2109(34.47)$ & 0.10 & \\
\hline 2019 & $358(10.63)$ & $2162(4.25)$ & 24.50 & & $284(9.28)$ & $540(8.83)$ & 1.59 & \\
\hline \multicolumn{9}{|l|}{ Race, $n(\%)$} \\
\hline $\begin{array}{l}\text { African } \\
\text { American }\end{array}$ & $549(16.31)$ & $8409(16.53)$ & 0.61 & 0.01 & $498(16.28)$ & $1000(16.35)$ & 0.18 & 0.89 \\
\hline Asian & $36(1.07)$ & $402(0.79)$ & 2.91 & & $30(0.98)$ & $71(1.16)$ & 1.75 & \\
\hline Caucasian & $2669(79.27)$ & $39,849(78.33)$ & 2.29 & & $2422(79.18)$ & $4827(78.90)$ & 0.68 & \\
\hline $\begin{array}{l}\text { Other/ } \\
\text { unknown }\end{array}$ & $113(3.36)$ & $2212(4.35)$ & 5.16 & & $109(3.56)$ & $220(3.60)$ & 0.18 & \\
\hline \multicolumn{9}{|l|}{ Ethnicity, $n(\%)$} \\
\hline Hispanic & $95(2.82)$ & $1810(3.56)$ & 4.19 & 0.03 & $86(2.81)$ & $173(2.83)$ & 0.10 & 0.99 \\
\hline Not Hispanic & 3097 (91.98) & $46,696(91.79)$ & 0.70 & & $2822(92.25)$ & $5647(92.30)$ & 0.18 & \\
\hline Unknown & $175(5.20)$ & $2366(4.65)$ & 2.53 & & $151(4.94)$ & $298(4.87)$ & 0.30 & \\
\hline \multicolumn{9}{|c|}{ Geographical region, $n(\%)$} \\
\hline Midwest & $1349(40.07)$ & $23,556(46.30)$ & 12.62 & $<0.0001$ & $1268(41.45)$ & $2543(41.57)$ & 0.23 & 0.98 \\
\hline Northeast & $512(15.21)$ & $6533(12.84)$ & 6.81 & & $474(15.50)$ & $943(15.41)$ & 0.23 & \\
\hline South & $1326(39.38)$ & $15,447(30.36)$ & 19.01 & & $1146(37.46)$ & $2290(37.43)$ & 0.07 & \\
\hline West & $99(2.94)$ & $3917(7.70)$ & 21.33 & & $96(3.14)$ & $202(3.30)$ & 0.93 & \\
\hline $\begin{array}{l}\text { Other/ } \\
\text { unknown }\end{array}$ & $81(2.41)$ & $1419(2.79)$ & 2.41 & & $75(2.45)$ & $140(2.29)$ & 1.07 & \\
\hline
\end{tabular}

$A C E i$ angiotensin-converting enzyme inhibitor, $A R B$ angiotensin receptor blocker, $S A C / V A L$ sacubitril/valsartan, $S D$ standard deviation, $S M D$ standardized mean difference 
New SAC/VAL user cohort

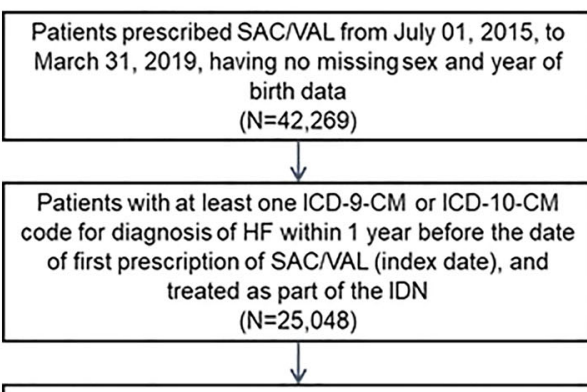

Patients with the first date active in a plan 365 days before the index date and last date active in a plan on the index date or beyond the index date* $(\mathrm{N}=22,273)$

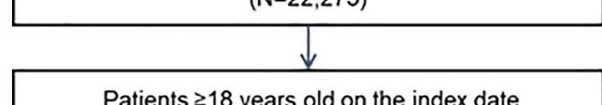

Patients $\geq 18$ years old on the index date $(\mathrm{N}=22,267)$

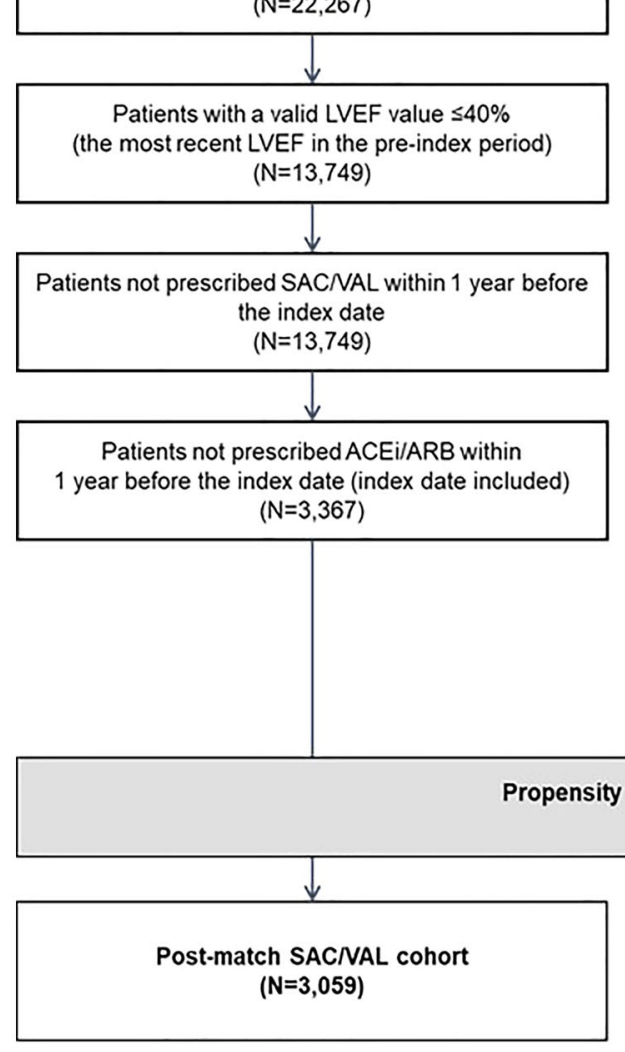

Fig. 2 Patient selection. ${ }^{*}$ Criteria used as a proxy for the continuous enrollment in the Optum EHR database as this information was not directly available. $A C E i$ angiotensin-converting enzyme inhibitor, $A R B$ angiotensin receptor blocker, $C M$ Clinical Modification, $E H R$

\section{New ACEi/ARB user cohort}

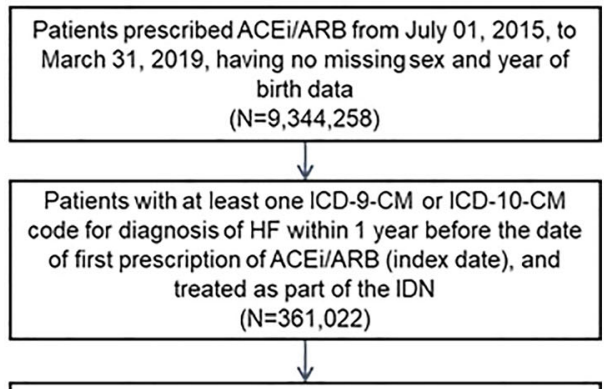

Patients with the first date active in a plan 365 days before the index date and last date active in a plan on the index date or beyond the index date* $(\mathrm{N}=319,119)$

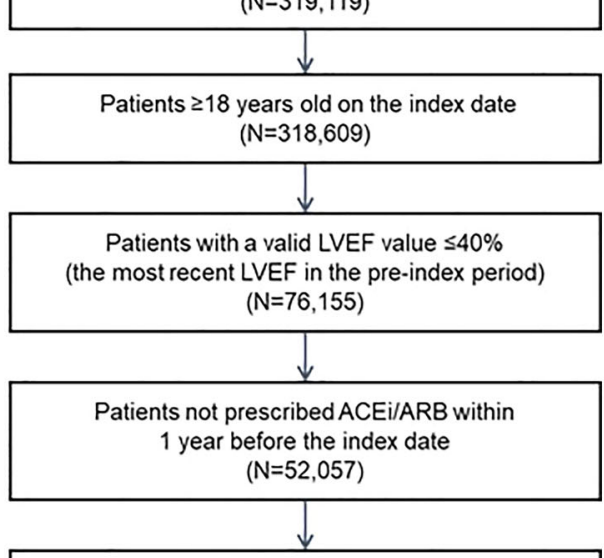

Patients not prescribed SACNAL within 1 year before the index date (index date included) $(\mathrm{N}=51,153)$

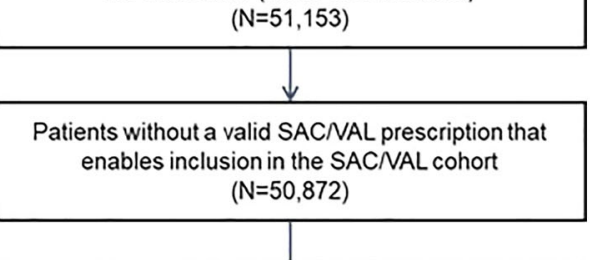

matching

$1: 2$

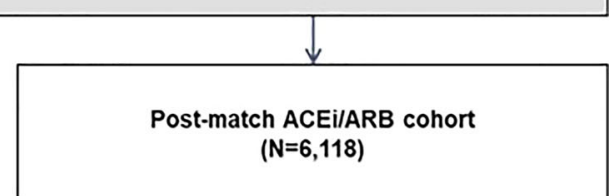

electronic health record, $H F$ heart failure, ICD International Classification of Diseases, IDN Integrated Delivery Network, $L V E F$ left ventricular ejection fraction, $S A C /$ $V A L$ sacubitril/valsartan 
Table 3 Distribution of comorbidities, signs, and symptoms before and after propensity score matching

\begin{tabular}{|c|c|c|c|c|c|c|c|c|}
\hline \multirow[t]{2}{*}{ Characteristics } & \multicolumn{4}{|c|}{ Before propensity score matching } & \multicolumn{4}{|c|}{ After propensity score matching } \\
\hline & $\begin{array}{l}\text { New SAC/ } \\
\text { VAL users } \\
(N=3367)\end{array}$ & $\begin{array}{l}\text { New ACEi/ } \\
\text { ARB users } \\
(N=50,872)\end{array}$ & $\begin{array}{l}\text { SMD } \\
(\%)\end{array}$ & $P$ value & $\begin{array}{l}\text { New SAC/ } \\
\text { VAL users } \\
(N=3059)\end{array}$ & $\begin{array}{l}\text { New } \\
\text { ACEi/ARB } \\
\text { users } \\
(N=6118)\end{array}$ & $\begin{array}{l}\text { SMD } \\
(\%)\end{array}$ & $P$ value \\
\hline \multicolumn{9}{|l|}{$\mathrm{ECI}^{\mathrm{a}}, n(\%)$} \\
\hline Low ECI score $<0$ & $9(0.27)$ & $205(0.40)$ & 2.35 & - & $9(0.29)$ & $33(0.54)$ & 3.81 & - \\
\hline Mild ECI score 0 & $7(0.21)$ & $220(0.43)$ & 3.98 & & $5(0.16)$ & $41(0.67)$ & 7.87 & \\
\hline $\begin{array}{l}\text { Moderate ECI score } \\
1-4\end{array}$ & $87(2.58)$ & $1540(3.03)$ & 2.68 & & $83(2.71)$ & $203(3.32)$ & 3.54 & \\
\hline Severe ECI score $\geq 5$ & $\begin{array}{l}3264 \\
\quad(96.94)\end{array}$ & $\begin{array}{l}48,907 \\
\quad(96.14)\end{array}$ & 4.40 & & $\begin{array}{l}2962 \\
\quad(96.83)\end{array}$ & $\begin{array}{l}5841 \\
\quad(95.47)\end{array}$ & 7.06 & \\
\hline \multicolumn{9}{|c|}{ Comorbidities, signs and symptoms, $n$ (\%) } \\
\hline Altered consciousness & $100(2.97)$ & $3699(7.27)$ & 19.61 & $<0.0001$ & $99(3.24)$ & $171(2.80)$ & 2.58 & 0.24 \\
\hline $\begin{array}{l}\text { Anemia (including } \\
\text { iron deficiency) }\end{array}$ & $204(6.06)$ & $4319(8.49)$ & 9.37 & $<0.0001$ & $199(6.51)$ & $416(6.80)$ & 1.18 & 0.60 \\
\hline COPD & $630(18.71)$ & $\begin{array}{l}11,525 \\
(22.65)\end{array}$ & 9.75 & $<0.0001$ & $594(19.42)$ & $\begin{array}{l}1128 \\
(18.44)\end{array}$ & 2.50 & 0.26 \\
\hline Dementia & $66(1.96)$ & $3115(6.12)$ & 21.26 & $<0.0001$ & $65(2.12)$ & $124(2.03)$ & 0.69 & 0.76 \\
\hline Depression & $342(10.16)$ & $6362(12.51)$ & 7.41 & $<0.0001$ & $322(10.53)$ & $588(9.61)$ & 3.04 & 0.17 \\
\hline Diabetes mellitus & $\begin{array}{l}1202 \\
(35.70)\end{array}$ & $\begin{array}{l}18,990 \\
\quad(37.33)\end{array}$ & 3.39 & 0.06 & $\begin{array}{l}1109 \\
(36.25)\end{array}$ & $\begin{array}{l}2178 \\
\quad(35.60)\end{array}$ & 1.36 & 0.54 \\
\hline $\begin{array}{l}\text { Dyslipidemia } \\
\text { (including } \\
\text { hypercholesterolemia) }\end{array}$ & $\begin{array}{l}2103 \\
\quad(62.46)\end{array}$ & $\begin{array}{l}31,221 \\
\quad(61.37)\end{array}$ & 2.24 & 0.21 & $\begin{array}{l}1907 \\
(62.34)\end{array}$ & $\begin{array}{l}3798 \\
\quad(62.08)\end{array}$ & 0.54 & 0.81 \\
\hline $\begin{array}{l}\text { Edema and fluid } \\
\text { overload }\end{array}$ & $404(12.00)$ & $6410(12.60)$ & 1.83 & 0.31 & $373(12.19)$ & $786(12.85)$ & 1.98 & 0.37 \\
\hline Hypertension & $\begin{array}{l}2339 \\
\quad(69.47)\end{array}$ & $\begin{array}{l}37,180 \\
\quad(73.09)\end{array}$ & 8.00 & $<0.0001$ & $\begin{array}{l}2157 \\
\quad(70.51)\end{array}$ & $\begin{array}{l}4255 \\
\quad(69.55)\end{array}$ & 2.11 & 0.34 \\
\hline Renal disease & $992(29.46)$ & $\begin{array}{l}14,921 \\
(29.33)\end{array}$ & 0.29 & 0.87 & $916(29.94)$ & $\begin{array}{l}1756 \\
(28.70)\end{array}$ & 2.73 & 0.22 \\
\hline Renal failure & $581(17.26)$ & $\begin{array}{l}11,901 \\
\quad(23.39)\end{array}$ & 15.30 & $<0.0001$ & $552(18.05)$ & $\begin{array}{l}1077 \\
\quad(17.60)\end{array}$ & 1.15 & 0.60 \\
\hline $\begin{array}{l}\text { Shortness of breath } \\
\text { (excluding sleep } \\
\text { apnea) }\end{array}$ & $\begin{array}{l}1362 \\
(40.45)\end{array}$ & $\begin{array}{l}21,056 \\
\quad(41.39)\end{array}$ & 1.91 & 0.28 & $\begin{array}{l}1249 \\
(40.83)\end{array}$ & $\begin{array}{l}2502 \\
\quad(40.90)\end{array}$ & 0.13 & 0.95 \\
\hline Sleep apnea & 619 (18.38) & $7898(15.53)$ & 7.62 & $<0.0001$ & $556(18.18)$ & $\begin{array}{l}1001 \\
\quad(16.36)\end{array}$ & 4.80 & 0.03 \\
\hline
\end{tabular}


Table 3 continued

\begin{tabular}{|c|c|c|c|c|c|c|c|c|}
\hline \multirow[t]{2}{*}{ Characteristics } & \multicolumn{4}{|c|}{ Before propensity score matching } & \multicolumn{4}{|c|}{ After propensity score matching } \\
\hline & $\begin{array}{l}\text { New SAC/ } \\
\text { VAL users } \\
(N=3367)\end{array}$ & $\begin{array}{l}\text { New ACEi/ } \\
\text { ARB users } \\
(N=50,872)\end{array}$ & $\begin{array}{l}\text { SMD } \\
(\%)\end{array}$ & $P$ value & $\begin{array}{l}\text { New SAC/ } \\
\text { VAL users } \\
(N=3059)\end{array}$ & $\begin{array}{l}\text { New } \\
\text { ACEi/ARB } \\
\text { users } \\
(N=6118)\end{array}$ & $\begin{array}{l}\text { SMD } \\
(\%)\end{array}$ & $P$ value \\
\hline \multicolumn{9}{|c|}{ Cardiac-specific comorbidities, signs, symptoms, and devices, $n$ (\%) } \\
\hline Angina pectoris & $264(7.84)$ & $3415(6.71)$ & 4.34 & 0.01 & $233(7.62)$ & $440(7.19)$ & 1.62 & 0.46 \\
\hline Atrial fibrillation & $\begin{array}{l}1398 \\
\quad(41.52)\end{array}$ & $\begin{array}{l}19,726 \\
\quad(38.78)\end{array}$ & 5.60 & 0.002 & $\begin{array}{l}1254 \\
\quad(40.99)\end{array}$ & $\begin{array}{l}2488 \\
\quad(40.67)\end{array}$ & 0.67 & 0.76 \\
\hline $\begin{array}{l}\text { Cardiac arrhythmia } \\
\text { (excluding atrial } \\
\text { fibrillation) }\end{array}$ & $\begin{array}{l}1140 \\
\quad(33.86)\end{array}$ & $\begin{array}{l}19,196 \\
(37.73)\end{array}$ & 8.09 & $<0.0001$ & $\begin{array}{l}1058 \\
\quad(34.59)\end{array}$ & $\begin{array}{l}2135 \\
\quad(34.90)\end{array}$ & 0.65 & 0.77 \\
\hline $\begin{array}{l}\text { Cardio- } \\
\text { resynchronization } \\
\text { therapy device }\end{array}$ & $\begin{array}{l}1226 \\
\quad(36.41)\end{array}$ & $\begin{array}{l}10,333 \\
\quad(20.31)\end{array}$ & 36.30 & $<0.0001$ & $\begin{array}{l}1010 \\
\quad(33.02)\end{array}$ & $\begin{array}{l}2011 \\
\quad(32.87)\end{array}$ & 0.31 & 0.89 \\
\hline $\begin{array}{l}\text { Cerebrovascular } \\
\text { disease }\end{array}$ & $349(10.37)$ & $7125(14.01)$ & 11.15 & $<0.0001$ & $327(10.69)$ & $639(10.44)$ & 0.80 & 0.72 \\
\hline $\begin{array}{l}\text { Ischemic heart disease } \\
\text { (including MI) }\end{array}$ & $\begin{array}{l}2240 \\
(66.53)\end{array}$ & $\begin{array}{l}34,331 \\
\quad(67.49)\end{array}$ & 2.04 & 0.25 & $\begin{array}{l}2047 \\
\quad(66.92)\end{array}$ & $\begin{array}{l}4000 \\
\quad(65.38)\end{array}$ & 3.25 & 0.14 \\
\hline $\begin{array}{l}\text { Peripheral artery } \\
\text { disease }\end{array}$ & $190(5.64)$ & $3561(7.00)$ & 5.58 & 0.003 & $180(5.88)$ & $378(6.18)$ & 1.24 & 0.58 \\
\hline $\begin{array}{l}\text { Peripheral vascular } \\
\text { disease }\end{array}$ & $329(9.77)$ & $5550(10.91)$ & 3.74 & 0.0396 & $306(10)$ & $592(9.68)$ & 1.10 & 0.62 \\
\hline Tachycardia & $824(24.47)$ & $\begin{array}{l}10,129 \\
(19.91)\end{array}$ & 11.00 & $<0.0001$ & $721(23.57)$ & $\begin{array}{l}1406 \\
(22.98)\end{array}$ & 1.39 & 0.53 \\
\hline Valvular heart disease & $\begin{array}{l}1475 \\
\quad(43.81)\end{array}$ & $\begin{array}{l}20,606 \\
\quad(40.51)\end{array}$ & 6.69 & 0.0002 & $\begin{array}{l}1331 \\
\quad(43.51)\end{array}$ & $\begin{array}{l}2661 \\
\quad(43.49)\end{array}$ & 0.03 & 0.99 \\
\hline
\end{tabular}

$A C E i$ angiotensin-converting enzyme inhibitor, $A R B$ angiotensin receptor blocker, $C O P D$ chronic obstructive pulmonary disease, $E C I$ Elixhauser Comorbidity Index, ICD International Classification of Diseases, $M I$ myocardial infarction, $S A C /$ $V A L$ sacubitril/valsartan, $S M D$ standardized mean difference

${ }^{a} \mathrm{ECI}$ categorizes the comorbidities based on ICD diagnosis codes. The ECI score ranges from -7 to 12 with higher scores indicating more severe symptom burden

were excluded from the analysis. For propensity matching, the most recent LVEF value (within the follow-up period) was used with hierarchical selection if multiple values were reported on the same day. All variables were included in the propensity score matching, regardless of the standardized mean difference (SMD) or $P$ value.
Categorical variables are summarized as frequency counts and percentages and compared using the Chi-squared test. Continuous variables are summarized as $n$, mean, and standard deviation (SD) and compared using an unequal variance two-sample $t$ test or the Mann-Whitney $U$ test (for continuous variables with skewed 
Table 4 Medication use before and after propensity score matching

\begin{tabular}{|c|c|c|c|c|c|c|c|c|}
\hline \multirow[t]{2}{*}{ Characteristics } & \multicolumn{4}{|c|}{ Before propensity score matching } & \multicolumn{4}{|c|}{ After propensity score matching } \\
\hline & $\begin{array}{l}\text { New SAC/ } \\
\text { VAL users } \\
(N=3367)\end{array}$ & $\begin{array}{l}\text { New ACEi/ } \\
\text { ARB users } \\
(N=50,872)\end{array}$ & $\begin{array}{l}\text { SMD } \\
(\%)\end{array}$ & $P$ value & $\begin{array}{l}\text { New SAC/ } \\
\text { VAL users } \\
(\boldsymbol{N}=3059)\end{array}$ & $\begin{array}{l}\text { New ACEi/ } \\
\text { ARB users } \\
(N=6118)\end{array}$ & $\begin{array}{l}\text { SMD } \\
\text { (\%) }\end{array}$ & $P$ value \\
\hline \multicolumn{9}{|l|}{ Medications, $n$ (\%) } \\
\hline $\begin{array}{l}\text { Aldosterone } \\
\text { antagonists }\end{array}$ & $939(27.89)$ & $9823(19.31)$ & 20.31 & $<0.0001$ & $817(26.71)$ & $1637(26.76)$ & 0.11 & 0.96 \\
\hline $\begin{array}{l}\text { Antiarrhythmics } \\
\text { and digoxin }\end{array}$ & $1792(53.22)$ & $33,856(66.55)$ & 27.45 & $<0.0001$ & $1704(55.7)$ & $3323(54.32)$ & 2.79 & 0.21 \\
\hline Beta-blockers & $995(29.55)$ & $22,885(44.99)$ & 32.33 & $<0.0001$ & $966(31.58)$ & $1874(30.63)$ & 2.05 & 0.35 \\
\hline $\begin{array}{l}\text { Calcium channel } \\
\text { blockers }\end{array}$ & $400(11.88)$ & $12,072(23.73)$ & 31.35 & $<0.0001$ & $397(12.98)$ & $841(13.75)$ & 2.26 & 0.31 \\
\hline $\begin{array}{l}\text { Lipid-lowering } \\
\text { drugs }\end{array}$ & $1636(48.59)$ & $30,346(59.65)$ & 22.34 & $<0.0001$ & $1552(50.74)$ & $3101(50.69)$ & 0.10 & 0.96 \\
\hline Loop diuretics & $2070(61.48)$ & $33,214(65.29)$ & 7.92 & $<0.0001$ & $1924(62.9)$ & $3782(61.82)$ & 2.23 & 0.32 \\
\hline $\begin{array}{l}\text { Mineralocorticoid } \\
\text { receptor } \\
\text { antagonists }\end{array}$ & $1105(32.82)$ & $13,388(26.32)$ & 14.28 & $<0.0001$ & $967(31.61)$ & $1974(32.27)$ & 1.40 & 0.53 \\
\hline Nitroglycerin & $1700(50.49)$ & $31,938(62.78)$ & 24.99 & $<0.0001$ & $1614(52.76)$ & $3214(52.53)$ & 0.46 & 0.84 \\
\hline
\end{tabular}

$A C E i$ angiotensin-converting enzyme inhibitor, $A R B$ angiotensin receptor blocker, $S A C / V A L$ sacubitril/valsartan, $S M D$ standardized mean difference

data). No imputation was performed for missing data.

A bivariate analysis was applied to the primary endpoint. All rate-of-event endpoints were modeled using a negative binomial model and the results are expressed as incidence rate ratios (IRRs) and 95\% confidence intervals (CIs). Time-to-event outcomes were modeled using a subdistribution hazards model and the results are expressed as hazard ratios (HRs) and 95\% CIs. The rate of HF-specific hospitalizations has significant competing risks of death and other hospitalizations. Therefore, a subdistribution hazards model was chosen over a Cox model as the former enables estimation of the incidence of event occurrence while taking competing risks into account, whereas the latter often overpredicts risk in scenarios with competing risks. For the purposes of this study, death and hospitalizations that were not of interest for any specific objective were accounted for as a competing risk within the subdistribution hazards model. The reliability of the results was assessed by replicating the results in both unadjusted and adjusted models. Variables that were significantly different between the cohorts after propensity score matching (i.e., those with an SMD $\geq 10 \%$ or $P<0.1$ ) were further used for adjustment in the model for a perfectly matched sample.

\section{RESULTS}

\section{Patient Characteristics}

Overall, 3367 new SAC/VAL users and 50,872 new ACEi/ARB users met the study selection criteria (Fig. 2). The two cohorts differed in several patient characteristics before matching. 
Table 5 Disease characteristics before and after propensity score matching

\begin{tabular}{|c|c|c|c|c|c|c|c|c|}
\hline \multirow[t]{2}{*}{ Characteristics } & \multicolumn{4}{|c|}{ Before propensity score matching } & \multicolumn{4}{|c|}{ After propensity score matching } \\
\hline & $\begin{array}{l}\text { New SAC/ } \\
\text { VAL users } \\
(N=3367)\end{array}$ & $\begin{array}{l}\text { New ACEi/ } \\
\text { ARB users } \\
(N=50,872)\end{array}$ & $\begin{array}{l}\text { SMD } \\
(\%)\end{array}$ & $P$ value & $\begin{array}{l}\text { New SAC/ } \\
\text { VAL users } \\
(N=3059)\end{array}$ & $\begin{array}{l}\text { New ACEi/ } \\
\text { ARB users } \\
(N=6118)\end{array}$ & $\begin{array}{l}\text { SMD } \\
(\%)\end{array}$ & $P$ value \\
\hline $\begin{array}{l}\operatorname{LVEF}(\%), \\
\text { mean }(\mathrm{SD})\end{array}$ & $26.69(8.15)$ & $29.21(8.21)$ & 30.76 & $<0.0001$ & $27.06(8.1)$ & $27.06(8.44)$ & 0.12 & 0.84 \\
\hline \multicolumn{9}{|c|}{ Previous HF hospitalizations, $n$ (\%) } \\
\hline 0 & $2534(75.26)$ & $44,243(86.97)$ & 30.26 & $<0.0001$ & $2395(78.29)$ & $4839(79.09)$ & 1.96 & 0.69 \\
\hline 1 & $543(16.13)$ & $4891(9.61)$ & 19.54 & & $454(14.84)$ & $898(14.68)$ & 0.46 & \\
\hline 2 & $153(4.54)$ & $1096(2.15)$ & 13.31 & & $114(3.73)$ & $212(3.47)$ & 1.40 & \\
\hline 3 & $64(1.90)$ & $322(0.63)$ & 11.35 & & $44(1.44)$ & $69(1.13)$ & 2.76 & \\
\hline$\geq 4$ & $73(2.17)$ & $320(0.63)$ & 13.13 & & $52(1.7)$ & $100(1.63)$ & 0.51 & \\
\hline \multicolumn{9}{|c|}{ Previous all-cause hospitalizations, $n$ (\%) } \\
\hline 0 & $1754(52.09)$ & $30,097(59.16)$ & 14.26 & $<0.0001$ & $1652(54)$ & $3337(54.54)$ & 1.08 & 0.87 \\
\hline 1 & $739(21.95)$ & $11,109(21.84)$ & 0.27 & & $678(22.16)$ & $1333(21.79)$ & 0.91 & \\
\hline 2 & $358(10.63)$ & $4392(8.63)$ & 6.78 & & $315(10.3)$ & $657(10.74)$ & 1.44 & \\
\hline 3 & $204(6.06)$ & $2170(4.27)$ & 8.11 & & $164(5.36)$ & $320(5.23)$ & 0.58 & \\
\hline$\geq 4$ & $312(9.27)$ & $3104(6.10)$ & 11.90 & & $250(8.17)$ & $471(7.70)$ & 1.75 & \\
\hline \multicolumn{9}{|c|}{ Previous HF-specific outpatient visits, $n$ (\%) } \\
\hline 0 & $587(17.43)$ & $20,368(40.04)$ & 51.58 & $<0.0001$ & $587(19.19)$ & $1157(18.91)$ & 0.71 & 0.99 \\
\hline 1 & $635(18.86)$ & $10,385(20.41)$ & 3.91 & & $609(19.91)$ & $1217(19.89)$ & 0.04 & \\
\hline 2 & $458(13.60)$ & $6137(12.06)$ & 4.60 & & $429(14.02)$ & $857(14.01)$ & 0.05 & \\
\hline 3 & $372(11.05)$ & $3777(7.42)$ & 12.54 & & $325(10.62)$ & $667(10.90)$ & 0.90 & \\
\hline$\geq 4$ & $1315(39.06)$ & $10,205(20.06)$ & 42.56 & & $1109(36.25)$ & $2220(36.29)$ & 0.07 & \\
\hline \multicolumn{9}{|c|}{ Previous HF-specific ER visits, $n$ (\%) } \\
\hline 0 & $3182(94.51)$ & $49,313(96.94)$ & 12.03 & $<0.0001$ & $2902(94.87)$ & $5852(95.65)$ & 3.69 & 0.34 \\
\hline 1 & $154(4.57)$ & $1356(2.67)$ & 10.23 & & $129(4.22)$ & $224(3.66)$ & 2.86 & \\
\hline 2 & $19(0.56)$ & $153(0.30)$ & 4.02 & & $19(0.62)$ & $33(0.54)$ & 1.08 & \\
\hline 3 & $4(0.12)$ & $32(0.06)$ & 1.86 & & $4(0.13)$ & $5(0.08)$ & 1.51 & \\
\hline$\geq 4$ & $8(0.24)$ & $18(0.04)$ & 5.48 & & $5(0.16)$ & $4(0.07)$ & 2.90 & \\
\hline
\end{tabular}

$A C E i$ angiotensin-converting enzyme inhibitor, $A R B$ angiotensin receptor blocker, $E R$ emergency room, $H F$ heart failure, $L V E F$ left ventricular ejection fraction, $S A C / V A L$ sacubitril/valsartan, $S M D$ standardized mean difference

Notably, compared with new ACEi/ARB users, new SAC/VAL users were younger; had a higher proportion of men (Table 2); a higher proportion of patients with tachycardia and cardiac resynchronization therapy; a lower proportion of patients with cerebrovascular 


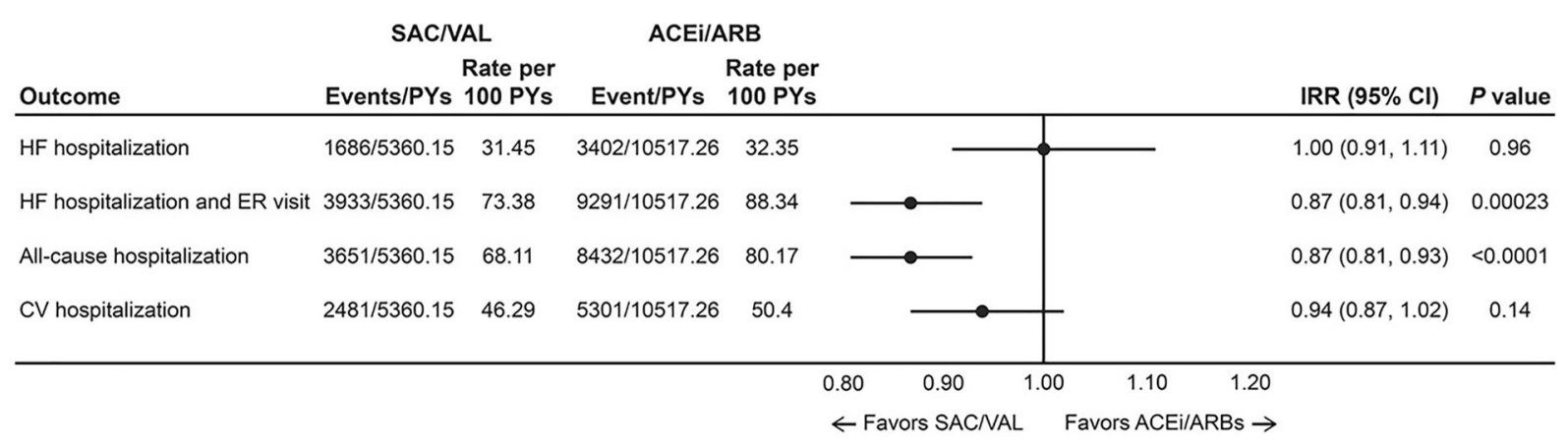

Fig. 3 Comparison of rate of events between new SAC/ VAL users and new ACEi/ARB users. $A C E i$ angiotensinconverting enzyme inhibitor, $A R B$ angiotensin receptor blocker, $C I$ confidence interval, $C V$ cardiovascular, $E R$ emergency room, $H F$ heart failure, IRR incidence rate ratio, $P Y$ patient-year, $S A C / V A L$ sacubitril/valsartan

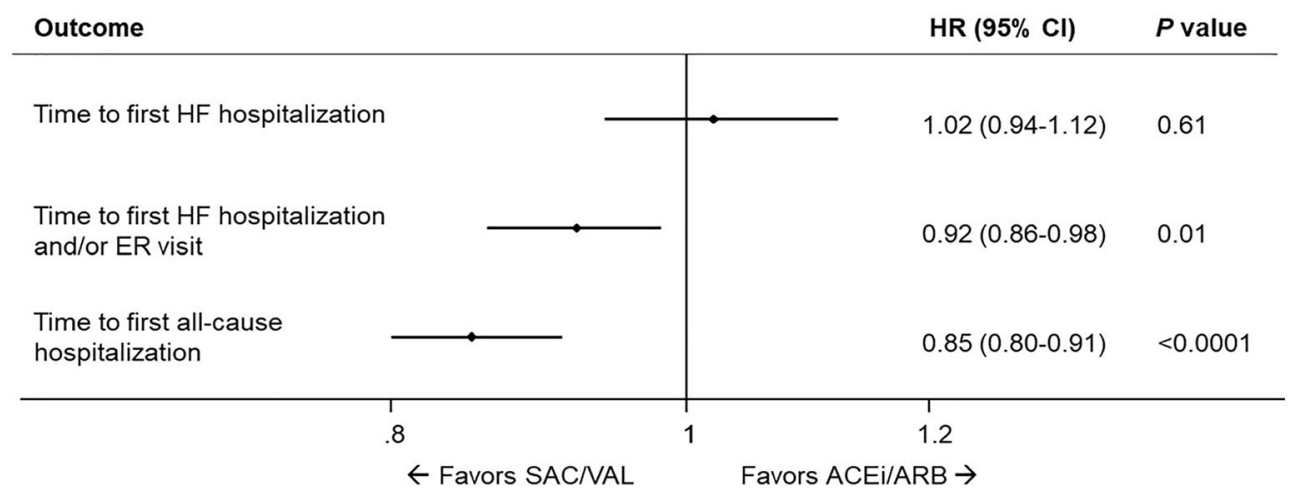

Fig. 4 Comparison of time to event between new SAC/ VAL users and new ACEi/ARB users. $A C E i$ angiotensinconverting enzyme inhibitor, $A R B$ angiotensin receptor

disease, renal failure, dementia, and altered consciousness (Table 3); a lower proportion of patients with prior medication use (except for aldosterone antagonists and mineraloreceptor antagonists, Table 4); a lower mean LVEF; and a higher proportion of patients with HF hospitalizations, all-cause hospitalizations, HF-specific outpatient visits, and HF-specific ER visits in the previous year suggestive of more severe HF in the SAC/VAL cohort (Table 5).

Propensity score matching could be performed for $91 \%$ of new SAC/VAL users and $12 \%$ of new ACEi/ARB users. After propensity score matching, there were 3059 patients in the SAC/ VAL cohort and 6118 patients in the ACEi/ARB cohort, with no significant differences between blocker, $C I$ confidence interval, $E R$ emergency room, $H F$ heart failure, $H R$ hazard ratio, $S A C / V A L$ sacubitril/valsartan

the two cohorts for any of the baseline characteristics except the proportion of patients with sleep apnea, which was higher in the SAC/VAL cohort (18.2 vs. $16.4 \% ; P=0.03)$. Baseline characteristics of the two groups after propensity score matching are presented in Tables 2,3 , 4 and 5 . More than $80 \%$ of patients had at least one HF-specific outpatient visit and approximately $46 \%$ of patients had at least one all-cause hospitalization during the 365-day pre-index period. Approximately $21 \%$ of patients had at least one HF hospitalization during the 365-day pre-index period, whereas $5 \%$ had at least one HF-specific ER visit. 


\section{Post-index Hospitalization}

The rates of events during the follow-up period are summarized in Fig. 3. The rate of post-index HF hospitalizations was similar between new SAC/VAL users and new ACEi/ARB users (31.45 per 100 person-years [PYs] vs. 32.35 per 100 PYs, respectively; IRR 1.00; 95\% CI 0.91-1.11; $P=0.96)$. The rate of all-cause hospitalization was $13 \%$ lower for new SAC/VAL users compared with new ACEi/ARB users (68.11 per 100 PYs vs. 80.17 per 100 PYs; IRR 0.87; 95\% CI 0.81-0.93; $P<0.0001)$. Similarly, the rate of the composite of HF hospitalization or ER visits was $13 \%$ lower for new SAC/VAL users compared with new ACEi/ARB users (73.38 per 100 PYs vs. 88.34 per 100 PYs; IRR 0.87; 95\% CI 0.81-0.94; $P=0.00023)$. The rate of cardiovascular hospitalizations was similar between the two cohorts (46.29 per 100 PYs vs. 50.40 per 100 PYs; IRR $0.94 ; 95 \%$ CI $0.87-1.02 ; P=0.14$ ).

The time-to-event analysis showed similar results, with new SAC/VAL users having a significantly lower risk of first HF hospitalization or ER visit (HR 0.92; 95\% CI 0.86-0.98; $P=0.01$ ) and first all-cause hospitalization (HR $0.85 ; 95 \%$ CI $0.80-0.91 ; P<0.0001)$ compared with new ACEi/ARB users (Fig. 4). The results were similar to those estimated using the unadjusted model (results not presented).

\section{DISCUSSION}

The new SAC/VAL users in this study had significantly lower rates of all-cause hospitalizations and the composite of HF hospitalization or ER visits than the new ACEi/ARB users; this was true in the analyses of both total rates and time to first event. These results were similar to those observed in a systematic literature review of real-world studies, wherein studies comparing SAC/VAL with ACEi/ARB in patients with HFrEF (previously treated or naïve to ACEi/ARB) reported a significantly lower risk of all-cause hospitalization with SAC/VAL [20].

Despite significant differences in the rate of all-cause hospitalization, the rates of $\mathrm{HF}$ and cardiovascular hospitalizations were similar between new SAC/VAL users and new ACEi/ARB users-an initially unexpected and clinically counterintuitive finding. However, another USbased real-world study by Tan et al. [17] involving patients with systolic HF observed a similar pattern-a significantly lower risk for allcause hospitalization in the SAC/VAL group than in the ACEi/ARB group $(P<0.001)$, but not for HF hospitalization $(P=0.26)$. Tan et al. considered that differences in coding practices could have influenced the analysis of HF-specific hospitalizations. As was done in the Tan et al. study, we also defined HF hospitalization per ICD-9-CM and ICD-10-CM codes for HF listed within the primary diagnoses associated with a hospitalization. Although the rate of HF hospitalizations was similar between treatment groups, a composite endpoint of HF hospitalization or ER visits did show a significant difference between SAC/VAL and ACEi/ARB, indicating that ACEi/ARB-treated patients are more likely to present to the ER for their HF than SAC/VAL-treated patients. Analyzing emergency visits that do not result in a hospitalization in addition to hospital admissions, may provide a complete picture of the burden of HF-related events to the healthcare system rather than focusing on hospitalizations alone. Moreover, all-cause hospitalization is considered more important by payers and patients than cause-specific hospitalization, as it represents the overall disease burden [21].

Frequent hospitalizations pose a major burden on patients with HF and their caregivers. In addition, consistent evidence indicates that the economic burden of HF is dominated by the hospitalization costs [11, 16, 22, 23]. A US-based analysis found that inpatient costs accounted for $80 \%$ of the total lifetime costs in HF [24]. Therefore, we expect that the reduction in allcause hospitalizations observed among the new SAC/VAL users in our study will be meaningful in reducing the overall burden of hospitalizations for patients. Reducing the risk of total hospitalizations due to any cause is of particular importance in the context of the current coronavirus disease 2019 (COVID-19) pandemic, when it is imperative to keep patients out of hospital.

To the best of our knowledge, the present study is the first real-world study to specifically 
compare SAC/VAL and ACEi/ARB use in patients with HFrEF naïve to prior RAASi therapy. Previous subgroup analyses have shown that the benefit of SAC/VAL is similar in patients naïve to ACEi/ARB and those pretreated with ACEi/ARB [9, 17]. A subgroup analysis of a US administrative claims database study showed a similar reduction in all-cause hospitalization with SAC/VAL in patients who were previously treated with ACEi/ARB and those naïve to ACEi/ARB [17]. In a sub analysis of the PIONEER-HF trial, the superiority of SAC/ VAL over enalapril was consistent in patients with or without prior exposure to ACEi/ARB $[9,10]$. The time to onset of HF hospitalization for the PIONEER-HF trial and the present realworld study were different (PIONEER-HF: HR 0.61, 95\% CI 0.40, 0.93; present study: HR 0.92, $95 \%$ CI 0.80, 0.91). However, it is important to note that time frame investigated in the present study is much longer than the 8-week time frame in PIONEER-HF trial. Due to the nature of the data and the varying index dates in the present study, we defined the follow-up period as until end of the study period (March 31, 2020), death or patient transfer out of the database. Therefore, in the present study, patients could potentially be assessed for time to HF hospitalization over a period greater than 3 years.

Our findings of the real-world benefit of SAC/VAL on all-cause hospitalizations in patients naïve to prior RAASi therapy are consistent with the results from these previous subgroup analyses and support the use of SAC/ VAL in this patient population. Moreover, the recent update of the ACC ECDP for optimization of HF treatment has now recommended a "direct-to-ARNI approach," that is to use ARNI as a de novo RAASi therapy in patients naïve to ACEi/ARB therapy [6].

The findings of the present study should be interpreted in light of several limitations. Because of the retrospective, observational nature of the study, the causal relationships between the clinical outcomes and the treatment could not be inferred. Medication use might have been overestimated because the drug prescription was used as a proxy for drug use. Therefore, it was not possible to determine whether the patients used their medication as prescribed. The use of SAC/VAL or ACEi/ARB may be influenced by the socioeconomic status of a patient. However, this parameter could not be adjusted as details on socioeconomic status were not available in Optum EHRs. Despite matching several key patient characteristics between the SAC/VAL and ACEi/ARB incident cohorts, a possibility of imbalances in the disease severity might exist, with a chance that patients with more persistent symptoms may have been prescribed SAC/VAL. Possibility of differences in the disease severity might be due to the non-availability of some of the variables, including NYHA class, blood pressure, and biomarkers (e.g., N-terminal pro-B-type natriuretic peptide and renal function markers). Another limitation was that the "naïve" populations in the study were defined as "being naïve to ACEi/ARB and SAC/VAL for at least 365 days before the initial prescription for either ACEi/ ARB or SAC/VAL". Although it was unlikely that patients were previously treated with SAC/VAL because of the overlapping of the identification period of our study with the date of licensing of SAC/VAL in the US, it could have been possible that an individual might not be "truly" naïve to ACEi/ARB and had merely not been prescribed ACEi/ARB during the 365 days before their index prescription within the database. Lastly, the results of this study may not be generalized to other populations, such as the new SAC/VAL and new ACEi/ARB users who were excluded from the analysis during propensity score matching, those with different ethnicity or from different regions, and those who were uninsured or on fee-for-service healthcare plans.

\section{CONCLUSIONS}

Use of SAC/VAL in RAASi-naïve patients with HFrEF resulted in lower rates of all-cause hospitalization and the composite of HF hospitalization or ER visits compared with ACEi/ARB treatment. The rates of $\mathrm{HF}$ and cardiovascular hospitalizations were similar between the two cohorts. These findings further strengthen the evidence base for SAC/VAL in ACEi/ARB-naïve 
patients. Initiating SAC/VAL directly in RAASinaïve patients with $\mathrm{HFrEF}$ can reduce total hospitalizations, thereby reducing the clinical and economic burden of HFrEF in these patients.

\section{ACKNOWLEDGEMENTS}

Funding. This study and the journal's Rapid Service Fee was funded by Novartis Pharma AG, Basel, Switzerland.

Authorship. All named authors meet the International Committee of Medical Journal Editors criteria for authorship for this article. All authors take responsibility for the integrity of the work as a whole, and have given their approval for this version to be published.

Authorship Contributions. All authors contributed to the study conception and design. Material preparation, data collection and analysis were performed by Sibasish Saha, Sarvesh Kumar Satwase and Emma Houchen. All authors read and approved the final manuscript.

Medical Writing, Editorial, and Other Assistance. Medical writing and editorial support for this manuscript was provided by Arushi Bamrara (Novartis Healthcare, Hyderabad) funded by Novartis.

Disclosures. Emma Houchen, Clare Proudfoot, Sibasish Saha, Sarvesh Kumar Satwase, and Rachel Studer are employees of Novartis. Emil Loefroth, Raymond Schlienger, and Stefano Corda were employees of Novartis at the time of this manuscript development and are presently associated with Copenhagen Economics, Galapagos, and Bayer. Clare Proudfoot and Rachel Studer hold shares of Novartis.

Compliance with Ethics Guidelines. Institutional review board approval was not required for this study because the information retrieved from the database was de-identified.
Data Availability. The data from the Optum EHR database are currently licensed and accessible within Novartis via the EVIdence of OutComes (EVICO) platform.

Prior Presentation. Part of this work has been previously presented in the American Heart Association 2020 conference.

Open Access. This article is licensed under a Creative Commons Attribution-NonCommercial 4.0 International License, which permits any non-commercial use, sharing, adaptation, distribution and reproduction in any medium or format, as long as you give appropriate credit to the original author(s) and the source, provide a link to the Creative Commons licence, and indicate if changes were made. The images or other third party material in this article are included in the article's Creative Commons licence, unless indicated otherwise in a credit line to the material. If material is not included in the article's Creative Commons licence and your intended use is not permitted by statutory regulation or exceeds the permitted use, you will need to obtain permission directly from the copyright holder. To view a copy of this licence, visit http://creativecommons.org/licenses/by$\mathrm{nc} / 4.0 /$.

\section{REFERENCES}

1. Virani SS, Alonso A, Aparicio HJ, et al. Heart disease and stroke statistics-2021 update: a report from the American Heart Association. Circulation. 2021;143(8):e254-743.

2. Lippi G, Sanchis-Gomar F. Global epidemiology and future trends of heart failure. AME Med J. 2020;5:15.

3. Seferovic PM, Ponikowski P, Anker SD, et al. Clinical practice update on heart failure 2019: pharmacotherapy, procedures, devices and patient management. An expert consensus meeting report of the Heart Failure Association of the European Society of Cardiology. Eur J Heart Fail. 2019;21(10): 1169-86.

4. Yancy CW, Jessup M, Bozkurt B, et al. 2017 ACC/ AHA/HFSA focused update of the 2013 ACCF/AHA guideline for the management of heart failure: a report of the American College of Cardiology/ 
American Heart Association Task Force on Clinical Practice Guidelines and the Heart Failure Society of America. J Cardiac Fail. 2017;23(8):628-51.

5. Fala L. Entresto (sacubitril/valsartan): first-in-class angiotensin receptor neprilysin inhibitor FDA approved for patients with heart failure. Am Health Drug Benefits. 2015;8(6):330-4.

6. Maddox TM, Januzzi JL Jr, Allen LA, et al. Update to the 2017 ACC expert consensus decision pathway for optimization of heart failure treatment: answers to 10 pivotal issues about heart failure with reduced ejection fraction: a report of the American College of Cardiology Solution Set Oversight Committee. J Am Coll Cardiol. 2021;77(6):772-810.

7. McMurray JJ, Packer M, Desai AS, et al. Angiotensinneprilysin inhibition versus enalapril in heart failure. N Engl J Med. 2014;371(11):993-1004.

8. Morrow DA, Velazquez EJ, DeVore AD, et al. Clinical outcomes in patients with acute decompensated heart failure randomly assigned to sacubitril/valsartan or enalapril in the PIONEER-HF trial. Circulation. 2019;139(19):2285-8.

9. Velazquez EJ, Morrow DA, DeVore AD, et al. Angiotensin-neprilysin inhibition in acute decompensated heart failure. N Engl J Med. 2019;380(6): 539-48.

10. Ambrosy AP, Braunwald E, Morrow DA, et al. Angiotensin receptor-neprilysin inhibition based on history of heart failure and use of renin-angiotensin system antagonists. J Am Coll Cardiol. 2020;76(9):1034-48.

11. Albert NM, Swindle JP, Buysman EK, Chang C. Lower hospitalization and healthcare costs with sacubitril/valsartan versus angiotensin-converting enzyme inhibitor or angiotensin-receptor blocker in a retrospective analysis of patients with heart failure. J Am Heart Assoc. 2019;8(9):e011089.

12. Chang C, Song X, Nguyen A, Smith D, Choi M, Duffy C. Hospitalizations of patients with heart failure and reduced ejection fraction treated with sacubitril/valsartan (S/V) vs. angiotensin-converting enzyme inhibitor or angiotensin receptor blocker (ACEI/ARB). Circ Cardiovas Qual Outcomes. 2018;11:A216.

13. Chang HY, Feng AN, Fong MC, et al. Sacubitril/valsartan in heart failure with reduced ejection fraction patients: real-world experience on advanced chronic kidney disease, hypotension, and dose escalation. J Cardiol. 2019;74(4):372-80.

14. Greene SJ, Lippmann SJ, Mentz RJ, Greiner MA, Hardy NC, Chang CL, Hammill BG, Luo N, Samsky MD, Heidenreich PA, Laskey WK, Yancy CW,
Peterson PN, Curtis LH, Hernandez AF, Fonarow GC, O'Brien EC. Clinical effectiveness of sacubitril/valsartan among patients hospitalized for heart failure with reduced ejection fraction. J Cardiac Fail. 2019;25(11):937.

15. Mohanty AF, Levitan EB, Dodson JA, et al. Characteristics and healthcare utilization among veterans treated for heart failure with reduced ejection fraction who switched to sacubitril/valsartan. Circ Heart Fail. 2019;12(11):e005691.

16. Parlett L, Gordan A, Sylvestrzak M, White J, Power $\mathrm{T}$, Barron J. Hospitalization rates and costs among real-world users of sacubitril/valsartan versus other heart failure medications. Circulation. 2018;138: A15928.

17. Tan NY, Sangaralingham LR, Sangaralingham SJ, et al. Comparative effectiveness of sacubitril-valsartan versus ACE/ARB therapy in heart failure with reduced ejection fraction. JACC Heart Fail. 2020;8(1):43-54.

18. Januzzi JL Jr, Prescott MF, Butler J, et al. Association of change in N-terminal Pro-B-type natriuretic peptide following initiation of sacubitril-valsartan treatment with cardiac structure and function in patients with heart failure with reduced ejection fraction. JAMA. 2019;322(11):1-11.

19. Wachter R, Fonseca AF, Balas B, et al. Real-world treatment patterns of sacubitril/valsartan: a longitudinal cohort study in Germany. Eur J Heart Fail. 2019;21(5):588-97.

20. Proudfoot C, Studer R, Rajput T, et al. Real-world effectiveness and safety of sacubitril/valsartan in heart failure: a systematic review. Int J Cardiol. 2021;331:164-71.

21. Joynt Maddox K, Bleser WK, Crook HL, et al. Advancing value-based models for heart failure: a call to action from the value in healthcare initiative's value-based models learning collaborative. Circ Cardiovasc Qual Outcomes. 2020;13(5): e006483.

22. Kilgore MPH, Kielhorn A, Maya JF, Sharma P. Economic burden of hospitalizations of Medicare beneficiaries with heart failure. Risk Manag Healthc Policy. 2017;10:63-70.

23. Urbich M, Globe G, Pantiri K, et al. A systematic review of medical costs associated with heart failure in the USA (2014-2020). Pharmacoeconomics. 2020;38(11):1219-36.

24. Dunlay SM, Shah ND, Shi Q, et al. Lifetime costs of medical care after heart failure diagnosis. Circ Cardiovasc Qual Outcomes. 2011;4(1):68-75. 